\title{
Current Development of Adjuvant Treatment of Non-Small-Cell Lung Cancer
}

\author{
Giorgio V. Scagliotti, Silvia Novello
}

\begin{abstract}
Although radical surgery remains the mainstay therapeutic modality for early-stage non-small-cell lung cancer (NSCLC), long-term survival of patients with completely resected NSCLC tumors remains suboptimal. Globally, the 5-year survival rate of patients who undergo complete surgical resection is in the range of $40 \%$ $\mathbf{5 0} \%$. The majority of postsurgical relapses are represented by distant metastases, with the risk of a local recurrence being $<10 \%$. Postoperative treatments, including chemotherapy, radiation therapy, or both, have been widely evaluated during recent decades. After almost 2 decades of disappointing results, the positive outcomes of 3 randomized studies have recently generated new hopes for a significant impact on survival by adjuvant chemotherapy. The 2 largest randomized studies of adjuvant chemotherapy in all stages (I-IIIA) of completely resected NSCLC that were adequately powered to detect small differences in survival yielded partially conflicting results but indicated that, if any benefit from adjuvant chemotherapy exists, it is approximately $\mathbf{5} \%$ at $\mathbf{5}$ years, as previously anticipated by a metaanalysis. More recently, 2 other randomized studies in selected subgroups of patients (one selectively performed in stage IB disease, the other in stage IB/II disease) indicate an unexpected significant benefit of approximately $15 \%$ at 5 years. Potential confounding factors may have contributed to such a significant benefit. A feature common to all these trials is the suboptimal therapeutic compliance to adjuvant chemotherapy, suggesting the need for careful selection of patients to be considered for adjuvant treatment. Genomic- and proteomic-driven chemotherapy as well as molecularly targeted therapies may represent additional areas of near-future clinical investigations.
\end{abstract}

Clinical Lung Cancer, Vol. 6, Suppl. 2, S63-S70, 2004

Key words: Chemotherapy, Metaanalysis, Molecular-targeted agents, Radiation therapy

\section{Introduction}

Surgery represents the main curative therapeutic approach for early-stage disease (stages IA-IIB) non-small-cell lung cancer (NSCLC). Unfortunately, these cases represent only a minority (20\%-25\%) of cases of NSCLC. Specific groups of patients with stage III disease also benefit from pulmonary resection, usually in combination with other treatment modalities. The use of a systemic therapy in completely resected NSCLC is reasonably justified by follow-up studies after radical resection that have shown predominance of distant failures over local recurrences and some clinical and pathologic evidence of early microdissemination of disease at the time of surgery.

Department of Clinical and Biological Sciences, University of Turin, S. Luigi Hospital, Thoracic Oncology Unit, Orbassano (Torino), Italy

Submitted: Oct 11, 2004; Revised: Nov 9, 2004; Accepted: Nov 12, 2004

Address for correspondence: Giorgio V. Scagliotti, MD, PhD,

Department of Clinical and Biological Sciences, University of Turin,

S. Luigi Hospital, Thoracic Oncology Unit, Regione Gonzole, 10,

10043 Orbassano (Torino), Italy

Fax: 39-011-903-8616; e-mail: giorgio.scagliotti@unito.it

\section{Pattern of Relapse After Complete} Resection for Early-Stage NSCLC

Long-term survival in NSCLC following surgical resection is stage-related, but even in stage IA, one third of patients will relapse and die of disease within 5 years. ${ }^{1}$ The majority of these relapses are distant metastases, with the risk of a local recurrence after complete resection being $<10 \%$. The brain is the most common site of metastatic recurrence, followed closely by bone, ipsilateral and contralateral lung, liver, and adrenals. More than $80 \%$ of recurrences occur within 2 years from the time of radical surgery. ${ }^{2}$

The rate of recurrence for patients with stage II disease is higher than in stage I; > 50\% of resected stage II disease can be expected to relapse and most recurrences are distant. The pattern of recurrence may differ by histology with more local recurrences seen for patients with squamous cell carcinoma and more distant metastases seen in patients with adenocarcinoma. ${ }^{3-6}$

\section{The Rationale for Adjuvant Treatments}

Following complete resection, tumor load, if any, is theoretically minimal. The relatively small number of residual neoplas-

Electronic forwarding or copying is a violation of US and International Copyright Laws. 
tic cells present in micrometastatic disease should contain few chemotherapy- or radiation-resistant clones. The Gompertzian model of tumor growth and regression fits experimental and clinical data of most human solid cancers - if the assumptions are effectively correct, when the tumor is clinically undetectable, its growth rate should be at its largest, and, although the numeric reduction induced by cytotoxic chemotherapy is small, the fractional cell kill from an effective dose of chemotherapy should be higher. ${ }^{7}$ In addition, pathologic staging allows better prediction of prognosis and facilitates the comparison of treatment results between different trials.

\section{Adjuvant Radiation Therapy}

For a long period of time, postoperative thoracic radiation therapy (RT) was the preferred adjuvant treatment. Results regarding its potential role have been reported from a large number of retrospective and prospective studies. Nine of these studies, collecting individual data from 2128 patients, have been included in the postoperative RT (PORT) metaanalysis and indicated PORT as a treatment with significant detrimental effect on survival. Data indicated a $21 \%$ relative increase in the risk of death, equivalent to an absolute detriment of $7 \%$ at 2 years, reducing the survival rate from $55 \%$ to $48 \%$ with PORT ( $P=0.001$; hazard ratio $[\mathrm{HR}], 1.21$; $95 \mathrm{CI}, 1.08-1.34$ ). Subset analyses suggested a trend toward greater negative effects for lower nodal status $(P=0.016$ for nodal status $0-2)$ and earlier stages of disease $(P=0.0003$ for stage I-III), with a distinct survival detriment for stage I disease and a clear lack of benefit or detriment for stage III disease. ${ }^{8}$

Most of the studies included in the PORT metaanalysis incorporated patients treated with older technology (cobalt 60) and different dosimetry, and these outdated parameters may be partially responsible for the higher mortality rate observed in the RT group attributable to an excess of intercurrent deaths. The use of newer technologies and improved dosimetry may prove to be effective, as more recently suggested in a retrospective review. ${ }^{9}$ In addition, in the PORT metaanalysis, there were no sufficient data on mediastinal lymph node dissection and the surgical procedure varied greatly among studies and centers. Consequently, the role of RT in patients with N2 disease remains unclear, as no definitive conclusions can be drawn from the PORT metaanalysis.

A study not included in the metaanalysis was subsequently reported and aimed to investigate the value of adjuvant postoperative external-beam RT in patients with radically resected NSCLC (pT13 pN0-2) compared with patients with resected NSCLC without adjuvant external-beam irradiation. In that study, 155 patients were randomized to surgery alone $(\mathrm{n}=72)$ or surgery followed by PORT $(\mathrm{n}=83)$. With a median follow-up of 3.6 years, there was no statistically significant difference in the overall 5-year survival rate (20.4\% with surgery alone vs. $29.7 \%$ with surgery plus PORT; $P>0.05)$. A subgroup analysis conducted separately for each pathologic stage did not result in any significant survival benefit for PORT when compared with surgery alone at 5 years ( $\mathrm{pN} 0$ and $\mathrm{pN} 1$ ) or 2 years (pN2). ${ }^{10}$ New randomized studies are awaited to assess the impact of adjuvant RT on local control and survival.
However, in consideration of the pattern of recurrence and relapse observed in patients who had previously completely resected early-stage NSCLC, it is quite unlikely that a local treatment such as postoperative RT - although it may potentially improve local control rate-will be able to significantly modify the survival of patients who have surgically resected early-stage NSCLC. Only the use of an effective systemic treatment that will eradicate micrometastatic clones has the potential to significantly affect survival. Theoretically, the combination of thoracic RT, delivered through the latest-generation RT machines, and platinum-based combination chemotherapy allows the optimization of local control and extrathoracic micrometastatic disease.

\section{History of Adjuvant Chemotherapy in Early-Stage NSCLC}

The history of adjuvant chemotherapy in completely resected NSCLC began in the early 1960s and 1970s with trials testing the roles of alkylating agents and nonspecific immunotherapies (mainly levamisole and Bacillus Calmette-Guérin) that uniformly failed to demonstrate any survival benefit; occasionally, a detrimental effect was observed. ${ }^{11}$ All the drugs used in these studies had shown very limited or no activity in advanced NSCLC.

How does one select the most appropriate treatment to be used in the adjuvant setting? Realistically, no definitive rules have been established, but the chosen treatment should at least be proven active in advanced-stage disease and be associated with good tolerability. ${ }^{12}$

In the 1980 s, the role of cisplatin-based combinations was extensively tested. These studies may be grouped into 2 series. Most of these studies included patients with predominantly stage III NSCLC, and CAP (cyclophosphamide/ doxorubicin/cisplatin) was more commonly investigated. All these studies failed to show any improvement in median and long-term survival. ${ }^{13-17}$

Two other studies testing the role of cisplatin-based chemotherapy were performed, mostly in patients with stage I disease. ${ }^{18,19}$ In one of these studies, 110 patients with completely resected T1 3N0 NSCLC were randomized to receive CAP or no additional therapy. After 10 years of follow-up, survival was significantly better in the experimental arm than in the control arm $(61 \%$ vs. $48 \%, P=0.05) .{ }^{18}$ In the second study, eligible patients with completely resected stage I NSCLC were classified by known prognostic factors and randomly assigned to receive 4 courses of CAP at 3-week intervals beginning on day 30 after surgery or no treatment. The CAP regimen consisted of 400 $\mathrm{mg} / \mathrm{m}^{2}$ cyclophosphamide, $40 \mathrm{mg} / \mathrm{m}^{2}$ doxorubicin, and 60 $\mathrm{mg} / \mathrm{m}^{2}$ cisplatin. Stratification by prognostic factors included histology, white blood cell count before surgery, and Karnofsky performance status before surgery. After a mean follow-up of 3.8 years, there were no differences in time to recurrence or overall survival (OS; not stratified by histology) between the 2 groups, even when analyses were adjusted for prognostic variables. ${ }^{19}$

Findings among these studies include variation in sample size, overestimation of the potential benefit of adjuvant chemotherapy, imbalance in patient and treatment characteristics (ie, incomplete mediastinal lymph node dissection), and, for a majority of these studies, impossibility of reaching the planned 
Talble 1 Recent Randomized Glinical Studies of Platinum Agent-Based Adjuvant Ghemotherapy in Completely Resected NSGI $6^{22-26}$

\begin{tabular}{|c|c|c|c|c|c|c|c|}
\hline Study & Site & $\begin{array}{c}\text { Disease } \\
\text { Stage }\end{array}$ & Chemotherapy Regimen & $\begin{array}{c}\text { Control } \\
\text { Arm }\end{array}$ & $\begin{array}{l}\text { Planned } \\
\text { Accrual }\end{array}$ & $\begin{array}{l}\text { Actual } \\
\text { Accrual }\end{array}$ & $\begin{array}{c}\text { Trial } \\
\text { Outcome }\end{array}$ \\
\hline ANITA I & International & I, II, IIIA & Cisplatin/Vinorelbine for 4 cycles & Observation & 800 & 831 & Pending \\
\hline Intergroup 0116 & United States & T1-3 N1/2 & Cisplatin/Etoposide for 4 cycles & RT & 462 & 488 & Negative \\
\hline JCOG & Japan & IIIA N2 & Cisplatin/Vindesine & Observation & 200 & 119 & Negative \\
\hline IALT $^{22 *}$ & International & I, II, IIIA & Cisplatin/Etoposide or Vinca Alkaloids for 3-4 cycles & Observation & 3300 & 1867 & Positive \\
\hline $\operatorname{ALPI}^{23 *}$ & Italy/Europe & I, II, IIIA & Cisplatin/Mitomycin C/Vindesine for 3 cycles & Observation & 1200 & 1209 & Negative \\
\hline BLT $^{24 *}$ & United Kingdom & I, II, IIIA & Cisplatin/Etoposide or Vinca Alkaloids for 3 cycles & Observation & 481 & 381 & Negative \\
\hline CALGB $9633^{25}$ & United States & IB & Carboplatin/Paclitaxel for 4 cycles & Observation & 504 & 344 & Positive \\
\hline NCIC BR. $10^{26}$ & Canada & T2 N0 T1/2 N1 & Cisplatin/Vinorelbine for 4 cycles & Observation & 640 & 482 & Positive \\
\hline
\end{tabular}

*Sequential thoracic RT allowed according to each institution policy; 13\% received RT in both arms of the study.

Abbreviations: ANITA = Adjuvant Navelbine International Trialist Association; BLT = Big Lung Trial; JCOG = Japanese Clinical Oncology Group

accrual. These flaws possibly reflect negative attitudes of thoracic surgeons toward adjuvant chemotherapy, and the modern multidisciplinary approach to patients with early-stage NSCLC may be a way to overcome this problem.

In addition, most of the trials' dose delivery, including total dose and dose intensity of chemotherapy agents, was often reported to be inadequate, with an average of $50 \%$ of patients receiving the full course of treatment.

\section{Recent Studies of Adjuvant Chemotherapy in NSCLC}

In 1995, a metaanalysis performed with different subgroups of patients with NSCLC receiving chemotherapy analyzed 8 cisplatin-based adjuvant chemotherapy trials in 1394 patients and demonstrated a $13 \%$ reduction of the risk of death, which was close to the borderline of statistical significance $(P=0.08)$. Similarly, there was a $6 \%$ reduction in the risk of death in patients treated with PORT and cisplatin-based chemotherapy compared with patients who received only PORT $(P=0.46)$. Conversely, adjuvant chemotherapy with long-term alkylating agents was significantly detrimental. ${ }^{20}$

These findings failed to affect clinical practice, not because the absolute gain was too small, but because such an estimate was still imprecise, ranging from a $1 \%$ detriment to a $10 \%$ benefit. In addition, the heterogeneity of surgical procedures and the difference in staging modalities strongly limit the applicability of the results of this metaanalysis. This was not the case, for example, for breast cancer adjuvant chemotherapy, the similarly narrow $6 \%$ benefit of which in 10-year survival rate emerged from a metaanalysis involving approximately 75,000 patients, with 31,000 recurrences and 24,000 deaths. ${ }^{21}$

This statistically insignificant benefit in 5-year survival of the previously mentioned NSCLC metaanalysis generated enough enthusiasm to prompt the planning of several randomized studies, all platinum agent-based (with or without thoracic RT) in completely resected NSCLC of stages I-IIIA (Table 1).22-26

The first study published was an Eastern Cooperative Oncology Group (ECOG) trial in which patients with clinical stage II/IIIA NSCLC after complete resection received RT alone or concurrent chemotherapy/RT. Overall toxicity was higher in the chemotherapy/RT group, but no difference was seen in efficacy outcomes. ${ }^{27}$ This study was criticized for the small sample size and for the absence of a pure control arm.

The studies in Table 1 are worthy of initial considerations. First, among these studies there is a huge difference in the sample size calculation, from $<500$ patients to $>3000$, to observe the same therapeutic effect in the same patient population. Second, some of these studies tested the role of adjuvant chemotherapy in all stages of resected NSCLC, whereas others addressed the same question to specific and well-defined subgroups of patients. Third, the only 2 trials designed to observe a reasonable survival advantage in the range of that previously described in the metaanalysis were the Adjuvant Lung Project Italy (ALPI) study and the International Adjuvant Lung Trial (IALT). ${ }^{22,23}$

The ALPI trial, conducted in 70 Italian centers and 7 European institutions affiliated with the European Organization for Research and Treatment of Cancer (EORTC), randomized patients surgically staged with stages I, II, and IIIA NSCLC to receive MVP (mitomycin $8 \mathrm{mg} / \mathrm{m}^{2}$ on day 1, vindesine 3 $\mathrm{mg} / \mathrm{m}^{2}$ on days 1 and 8 , and cisplatin $100 \mathrm{mg} / \mathrm{m}^{2}$ on day 1 every 3 weeks for 3 cycles) or no chemotherapy. ${ }^{23}$ Delivery of PORT (total dose 50-54 Gy in 5-6 weeks, beginning $\geq 4$ weeks after the completion of chemotherapy) was left to the policy of participating centers and randomization was stratified accordingly (Table 2). ${ }^{22,23}$

Between January 1994 and February 1998, 1209 patients were randomized. After a median follow-up of 64.5 months, differences in progression-free survival (PFS; HR, 0.89; $P=0.144$ ) and OS (HR 0.96; $P=0.585$ ) were not statistically significant. Only $69 \%$ of patients received the 3 full MVP cycles with or without dose adjustments or omissions. On multivariate analysis, only stage and sex emerged as independent prognostic factors. Moreover, there was no good evidence of differential effect of chemotherapy in the different subgroups of patients; in stage I, II, and IIIA disease, the HRs for survival were 0.97 (95\% CI, 0.71-1.33), 0.80 (95\% CI, 0.60-1.06) and 1.06 (95\% CI, 0.82-1.38), respectively (for interaction, $P=0.52$ ). Similar figures were found for PFS: HRs of 0.89 (95\% CI, 0.66-1.19), 0.78 (95\% CI, 0.60-1.03), and 0.94 (95\% CI, $0.73-1.21)$, respectively. It is remarkable to observe that, in the subgroup of patients with stage II NSCLC, although the HR 
Tahle 2 Patient Gharacteristics of the IMIT and AIPI Studies ${ }^{22,23}$

\begin{tabular}{|c|c|c|c|c|}
\hline \multirow[b]{2}{*}{ Characteristics } & \multicolumn{2}{|c|}{ IALT $^{22}$} & \multicolumn{2}{|c|}{$\mathrm{ALPI}^{23 *}$} \\
\hline & $\begin{array}{l}\text { Chemotherapy } \\
\qquad(\mathrm{n}=932)\end{array}$ & $\begin{array}{c}\text { Control } \\
(n=935)\end{array}$ & $\begin{array}{l}\text { Chemotherapy } \\
\qquad(n=472)\end{array}$ & $\begin{array}{l}\text { Control } \\
(n=465)\end{array}$ \\
\hline Median Age & 59 Years & 59 Years & 61 Years & 61 Years \\
\hline \multicolumn{5}{|l|}{ Pathologic Stage } \\
\hline I & $36 \%$ & $37 \%$ & $39 \%$ & $38 \%$ \\
\hline II & $25 \%$ & $24 \%$ & $31 \%$ & $34 \%$ \\
\hline IIIA & $39 \%$ & $39 \%$ & $29 \%$ & $28 \%$ \\
\hline \multicolumn{5}{|l|}{ Histology } \\
\hline Squamous cell & $46 \%$ & $47 \%$ & $51 \%$ & $49 \%$ \\
\hline Non-squamous cell & $54 \%$ & $53 \%$ & $49 \%$ & $51 \%$ \\
\hline Pneumonectomies & $35 \%$ & $35 \%$ & $24 \%$ & $26 \%$ \\
\hline Complete Lymph Node Dissection & \multicolumn{2}{|c|}{ Not Reported } & $55 \%$ & $53 \%$ \\
\hline Postoperative RT & \multicolumn{2}{|l|}{$30 \%$} & $43 \%$ & $43 \%$ \\
\hline Receiving Planned RT & $70.4 \%$ & $84.2 \%$ & $74 \%$ & $89 \%$ \\
\hline Median Follow-up Time & \multicolumn{2}{|c|}{56 Months } & \multicolumn{2}{|c|}{56 Months } \\
\hline
\end{tabular}

*From the final analysis, 108 patients from one center were excluded ( 54 in each arm) because of serious concerns about data integrity.

ascertain, as the choice of regimen was not stratified and patient case mix among centers could be extremely variable.

In the ALPI and IALT studies, relapse and recurrence of neoplastic disease accounted for the main cause of death and, more relevantly, in both arms of the ALPI study, > 40\% of patients had brain relapses.

Another feature common to both trials was the suboptimal compliance with adjuvant chemotherapy, with $8 \%$ and $9 \%$ of patients who never received chemotherapy and $26 \%-31 \%$ of patients who received less than the 3 planned courses of treatment, which compromised the relative dose intensity. In a comparative analysis of IALT and ALPI, delivery ability of chemotherapy did not differ dramatically when a doublet combination was used in-

was not statistically significant, a $10 \%$ survival advantage at 5 years for chemotherapy-treated patients was reported. ${ }^{23}$

In this trial, the choice for a triplet combination was suggested by the positive data reported from a trial comparing 3 chemotherapy regimens in patients with advanced NSCLC ${ }^{28}$ and by the efficacy of the MVP regimen in the neoadjuvant setting. ${ }^{29}$ In patients with stage IIIA NSCLC, triplet combinations were used as induction regimens in 2 small randomized phase III trials that showed a clinically meaningful superiority of the combined approach over surgery alone. ${ }^{30,31}$

The other large worldwide randomized trial, the IALT trial, was aimed at determining the impact on OS of a chemotherapy regimen including cisplatin $\left(80-120 \mathrm{mg} / \mathrm{m}^{2}\right)$ and a vinca alkaloid (vindesine $3 \mathrm{mg} / \mathrm{m}^{2}$ weekly, vinblastine $4 \mathrm{mg} / \mathrm{m}^{2}$ weekly, or vinorelbine $30 \mathrm{mg} / \mathrm{m}^{2}$ weekly) or etoposide $\left(100 \mathrm{mg} / \mathrm{m}^{2}\right.$ daily for 3 consecutive days) compared with no chemotherapy after complete surgical resection in patients with stage I-III NSCLC $^{22}$ (Table 2). ${ }^{22,23}$ Chemotherapy treatment was administered every 3-4 weeks. As in the ALPI trial, thoracic RT could be given according to the preregistration policy of each center. The planned number of patients was 3300 to observe a 5\% survival difference at 5 years (from $50 \%$ to $55 \%$ ). The study started in 1995 and was stopped in December 2000 because of slow accrual after enrolling 1867 patients. The median follow-up was 56 months. Compliance with chemotherapy was good; $74 \%$ of patients received $\geq 240 \mathrm{mg} / \mathrm{m}^{2}$ of cisplatin. Only $23 \%$ of patients on the chemotherapy arm experienced grade 4 toxicity. There was a toxic death rate of $0.8 \%$, which was attributed to the administration of chemotherapy. Disease-free survival and OS were increased by chemotherapy, with absolute survival benefits of $5.1 \%(P=0.003)$ and $4.1 \%(P=0.03)$, respectively. 22 However, a comparison of the risk-benefit profile of the different regimens used in this study is clearly difficult to stead of a triplet combination. Furthermore, the ALPI study showed that MVP conferred only a small, statistically nonsignificant, OS advantage even in the per-protocol exploratory analysis that compared outcomes among patients receiving all 3 planned cycles of chemotherapy with those of patients undergoing no adjuvant therapy.

Reasons for reduced therapeutic compliance may be related to the need for more time to fully recover from the surgical procedure itself for patients with lung cancer in comparison with the time needed for patients with breast cancer and may also be related to a negative selection bias of the patients enrolled in both studies. In the ALPI and IALT studies, $26 \%$ and 35\% of patients received pneumonectomies, a percentage far exceeding the normal pneumonectomy rate in any surgical series.

Data have been recently reported about adjuvant chemotherapy in a subgroup of patients with surgically resected disease enrolled in the British Big Lung Trial. Three hundred sixtyeight patients were randomized to receive cisplatin-based doublet (38\%) or triplet regimens (62\%). The reported HR for OS was 1.02 , but the limited sample size, quality of surgery, and limited follow-up period greatly reduce the power of the information provided. ${ }^{29}$

Different from ALPI and IALT, 2 additional adjuvant trials used third-generation chemotherapy regimens and were focused on a more restricted patient population. The results of the 2 trials were presented at the 40th Annual Meeting of the American Society of Clinical Oncology in 2004. Both studies were designed in the mid-1990s to compare adjuvant carboplatin/paclitaxel (Cancer and Leukemia Group B [CALGB] 9633 study) ${ }^{25}$ or cisplatin/vinorelbine (National Cancer Institute of Canada [NCIC] BR.10 study) ${ }^{26}$ with no adjuvant therapy for patients with completely resected stage IB disease (CALGB trial) or stage IB/II NSCLC (NCIC-BR.10; Table 3). ${ }^{25,26}$ 
The CALGB 9633 study accrued patients very slowly and, based on occurrence of events over time, the requested number of patients was amended to 384. It was well balanced for known prognostic factors between the treatment and control arms. An independent data monitoring committee stopped the study early at a planned interim analysis after the inclusion of $344 \mathrm{pa}-$ tients based on unequivocal superiority of one arm over the other.

The CALGB 9633 study demonstrates a remarkable improvement in OS compared with the no-treatment group (12\% at 4 years). However, it should be known that the median follow-up is only 34 months, and there is still a huge number of censored patients on both survival curves. The magnitude of benefit of the use of adjuvant carboplatin/paclitaxel was substantially greater than one might have predicted on the basis of IALT and the metaanalysis, and, considering the available data, an overestimation of the treatment effect appears reasonable.

Most notably, the delivery of chemotherapy was excellent in the treatment group, nearly $85 \%$ of patients in the treatment group received 4 cycles of chemotherapy. Toxicity in this group of patients was minimal, with only $36 \%$ of patients having grade 3/4 myelosuppression. There were no treatment-related deaths, which is an important aspect of an adjuvant study. Dose delays were uncommon, attesting to the well tolerated nature of the chemotherapy. ${ }^{25}$

It could be argued that the positive results were caused by a uniform patient population, a regimen that was well tolerated and nontoxic, and the fact that such a high fraction of patients was able to complete all the planned cycles of chemotherapy. The NCIC-BR.10 study randomized 482 patients with completely resected stage IB/II NSCLC to observation or 4 cycles of cisplatin/vinorelbine (Table 3). Cisplatin was given on days 1 and 8 , which allowed for better dose intensity. Overall survival was significantly improved (5-year survival, 69\% vs. 54\%; HR, $0.69 ; P=0.01)$ as well as PFS.

This regimen was less well tolerated than in the CALGB study, with the occurrence of grade $3 / 4$ neutropenia in $73 \%$ of the patients and febrile neutropenia in $6 \%$. Thirty-four percent of patients did not begin therapy or received only 1 cycle, and adjuvant treatment was terminated early for patient refusal in $30 \%$ of the cases and drug toxicity in $12 \%$ of patients. ${ }^{26}$ All together, these toxicity issues partially limit the applicability of such treatment in the daily practice.

Why were these 2 studies largely positive, exceeding so far the $5 \%$ benefit hypothesized by the metaanalysis and confirmed by the IALT study? Several potential confounding factors should be taken into consideration. First, each adjuvant study enrolled a select patient population (Table 4) 22,23,25,26; therefore, it is not known how much is representative of the entire population of patients with completely resected NSCLC. Second, in many of these studies no information is available about the proportion of patients who, during surgical resection, underwent systematic lymph nodal dissection or mediastinal lymph node sampling. In a recent randomized clinical study, systematic lymph nodal dissection was found to significantly influence survival in every stage of resectable NSCLC. 32 Third, patients with lung cancer frequently have comorbidities, including chronic obstructive pulmonary disease and cardiovascular diseases, that were found to significantly affect survival. ${ }^{33,34}$ Additionally, an imbalance in the proportion of patients who potentially quit smoking after radical surgery may account for survival differences. This is shown in a study of 273 patients with pathologic stage I NSCLC in which the amount of smoking exposure was found to be a highly significant predictor of OS. 35

\section{Uraci//Tegafur-Based Trials and Metaanalysis}

Several Japanese trials have evaluated the potential of oral fluorouracil derivatives as adjuvant treatments alone or in combination with other agents. Initially, 2 Japanese trials investigated the prolonged administration ( 6 months to 1 year) of uracil/tegafur (UFT; molar ratio of 1:4). Both trials considered eligible patients with completely resected NSCLC of stages I-III and showed a survival advantage for patients treated with UFT. In one study, patients were randomized to cisplatin, doxorubicin followed by

\begin{tabular}{|c|c|c|c|c|}
\hline Tahle 4 & \multicolumn{4}{|c|}{$\begin{array}{l}\text { Patient Enrollment of Randomized Studies of Adjuwant } \\
\text { Chemotherapy 22,23,25,26 }\end{array}$} \\
\hline \multicolumn{2}{|c|}{ Study } & $\begin{array}{l}\text { Enrollment } \\
\text { Period }\end{array}$ & $\begin{array}{l}\text { Enrolled } \\
\text { Patients }\end{array}$ & $\begin{array}{l}\text { Enrolled Patients } \\
\text { per Year }\end{array}$ \\
\hline \multicolumn{2}{|l|}{ IALT $^{22}$} & $1995-2000$ & 1867 & 339 \\
\hline \multicolumn{2}{|c|}{$\mathrm{ALPI}^{23}$} & 1994-1999 & 1209 & 242 \\
\hline \multicolumn{2}{|c|}{ CALGB $9633^{25}$} & $1996-2003$ & 344 & 49 \\
\hline \multicolumn{2}{|c|}{ NCIC BR.1026 } & $1995-2000$ & 482 & 80 \\
\hline
\end{tabular}


UFT, or observation; whereas in the other study, patients were randomly allocated to receive 1 cycle of cisplatin/vindesine followed by oral UFT for 1 year, oral UFT alone, or observation. ${ }^{36,37}$ In the first trial, survival advantage was shown only after adjustment in the imbalance for major prognostic factors, whereas in the second, postoperative chemotherapy was found to be a significant prognostic factor for survival. The overall 5-year survival rates were $64.1 \%$ for the UFT group $(\mathrm{n}=103)$ and $49 \%$ for the surgery-alone group $(\mathrm{n}=98 ; P=0.02)$.

A clinical study limited to 225 patients with stage I/II NSCLC reported on oral UFT with MVP as adjuvant chemotherapy. The 5-year survival rates were $71.1 \%$ for the surgery-alone group and $76.8 \%$ for the chemotherapy group, with no significant difference observed. A subset analysis showed prognostic advantage for the chemotherapy group. ${ }^{38}$ Another study randomized 221 patients with resected stage I/II NSCLC to UFT alone or control and failed to show any improvement in 5-year survival rate (79\% vs. $75 \%) .39$ At the beginning of 2004, a large confirmatory phase III trial of adjuvant UFT for 2 years versus control in resected stage I adenocarcinoma of the lung was reported. Stratification was based on age, sex, and pathologic stage (T1 vs. T2). Final results at 5 years showed a modest but significant OS benefit $(P=0.035)$ that was essentially confined to patients with T2 disease $(P=0.051) .40$ One questionable point in this trial is the absence of any advantage in disease-free survival (DFS) for the UFT-treated arm, which clearly contrasts with all the positive platinum agent-based adjuvant studies (IALT, 22 NCIC BR.10,25 CALGB 963326) in which improvement in OS for patients receiving adjuvant chemotherapy was invariably associated with a similar or greater magnitude of improvement in DFS.

Most of the previously mentioned trials have been included in a specific metaanalysis evaluating the efficacy of UFT alone, including 2003 patients globally, 90\% with stage I NSCLC. Patients treated with UFT had improvements in 5-year survival of $4.6 \%$ (HR, 0.77; 95\% CI, 0.63-0.94; $P=0.01$ ) and 7-year survival of 7\% (HR, 0.74; 95\% CI, 0.61-0.88; $P=0.001$ ). ${ }^{41}$

In most of these UFT studies, adjuvant treatment was planned for 2 consecutive years, and treatment compliance was generally higher than that observed in cisplatin-based studies. The concept of relatively mild, low-dose continuous adjuvant therapy is attractive, but the absence of confirmatory adjuvant UFT studies outside Japan strongly limit the applicability of these data into clinical practice because, even recently, randomized studies in second-line treatment for NSCLC with gefitinib, an epidermal growth factor receptor (EGFR) tyrosine kinase (TK) inhibitor, indicated a different activity of the drug when tested among Japanese and non-Japanese patients. ${ }^{42,43}$

\section{Molecularly Targeted Agents in the Adjuvant Setting}

There is a good rationale for the use of molecularly targeted therapies in early NSCLC, considering that many of the pathways these agents target have been shown to be altered in very early phases in the natural history of the disease. ${ }^{44}$ The smallmolecule TK inhibitor gefitinib inhibits EGFR, believed to promote tumor cell growth and metastases. A phase III randomized trial will test the effectiveness of gefitinib in the adjuvant setting for patients with curatively resected stage IB, II, or IIIA disease. The trial will compare OS for patients treated with daily gefitinib or placebo. Treatment in both arms will continue for 2 years in the absence of disease progression or unacceptable toxicity. The projected accrual is 1242 patients (621 per treatment arm), with patients stratified according to disease stage (stage IB vs. II vs. IIIA) and histologic subtype (squamous cell vs. other types). After publication of the IALT study of adjuvant cytotoxic chemotherapy, cisplatin-based therapy was considered a therapeutic option before the randomization of the patients, and it is now considered a stratification factor. The trial will also determine the prognostic significance of the EGFR expression level, phosphorylation, and mutations in the primary tumor.

\section{Molecular Prognostic Factors}

In $30 \%$ of lung adenocarcinomas and in approximately $10 \%$ of large-cell carcinomas, the K-ras gene was found to be mutationally activated, and in pivotal studies this feature was a prognostic determinant of survival, regardless of the stage of disease. 45 These data have not been fully confirmed by additional clinical studies. 46 The genetic alterations affecting the $p 53$ gene are among the most common changes that occur during malignant progression of several types of tumors, including NSCLC. However, studies that explored the prognostic role of $p 53$ mutations in NSCLC reported highly conflicting results and no definitive information could be obtained from those studies. ${ }^{47}$

The expression of $\mathrm{K}$-ras, $p 53$, and $\mathrm{Ki}-67$ was prospectively evaluated in subgroups of patients included in the ALPI trial; unfortunately, no relevant prognostic implication was found. ${ }^{23}$ The CALGB 8633 study also aimed at evaluating the prevalence of 10 molecular biologic markers (growth factors HER2/neu and $\mathrm{K}$-ras codon 12 mutations, cell-cycle factors $\mathrm{Ki}-67$ and retinoblastoma, apoptosis factors $p 53$ and $b c l-2$, angiogenesis factor VIII, and adhesion protein CD44, and motility factor gelsolin) to determine the influence of adjuvant chemotherapy on cancer-free survival relative to marker expression in these patients, but this information is not yet available. ${ }^{30}$ However, it should be noted that the largest biomarker study, retrospectively performed in 515 cases of resected stage I NSCLC, failed to show any significant association between survival and the expression of an extensive panel of biomarkers, including EGFR, HER2/neu, bcl-2, p53, and angiogenesis. ${ }^{48}$

\section{Neoadjuvant Studies in Early-Stage NSCLC}

The benefits of neoadjuvant chemotherapy have already been widely accepted in the setting of locally advanced marginally resectable (stage IIIA N2) and unresectable disease (stage IIIB). A pivotal phase III study of a French thoracic cooperative group compared the administration of 2 courses of mitomycin C/ifosfamide/cisplatin followed by surgery compared with surgery alone in resectable stages I-IIIA disease. Responding patients received additional chemotherapy after surgery. The response rate to induction therapy was $64 \%$ (11\% pathologic complete response). A survival advantage, potentially delayed for high perioperative toxicity, was observed in the combined arm. Median 


\begin{tabular}{l|c|c|c|}
\hline \multicolumn{3}{|c|}{ Tahle 5} & $\begin{array}{c}\text { Ongoing Neoadjuluant Studies in Resectahle } \\
\text { Non-Small-Gell Lung Gancer }\end{array}$ \\
\hline \multicolumn{1}{|c|}{ Study } & Induction Treatment & $\begin{array}{c}\text { Current } \\
\text { Accrual }\end{array}$ & $\begin{array}{c}\text { Planned } \\
\text { Accrual }\end{array}$ \\
\hline $\begin{array}{l}\text { US Intergroup } \\
\text { (SWOG 9900) }\end{array}$ & Carboplatin/Paclitaxel & 356 & 600 \\
\hline $\begin{array}{l}\text { MRC LU22/ } \\
\text { EORTC }\end{array}$ & $\begin{array}{c}\text { Mitomycin C/Vinblastine/Cisplatin, } \\
\text { Mitomycin C/Ifosfamide/Cisplatin, } \\
\text { Vinorelbine/Cisplatin }\end{array}$ & 450 & 600 \\
\hline ChEST & $\begin{array}{c}\text { Gemcitabine/Cisplatin } \\
\text { Carboplatin/Paclitaxel }\end{array}$ & 250 & 700 \\
\hline NATCH & $\begin{array}{c}\text { Carboplatin/Paclitaxel or } \\
\text { Gemcitabine/Cisplatin }\end{array}$ & 300 & 628 \\
\hline IFCT (France) & \multicolumn{2}{|c|}{520} \\
\hline
\end{tabular}

*The study was halted in July 2004 after the presentation of the data of the CALGB 9633 and NCIC BR.10 trials showing the positive impact of adjuvant chemotherapy. ${ }^{25,26}$

Abbreviations: IFCT $=$ Intergroupe Francophone de Cancérologie Thoracique; MRC $=$ Medical Research Council

survival time favored the combined approach (36 months vs. 26 months; $P=0.11$, log-rank test). After 150 days, the effect of perioperative chemotherapy on survival was significantly favorable (relative risk, $0.71 ; P=0.03$ ). A quantitative interaction between nodal stage and treatment was also noted, with the benefit from perioperative chemotherapy confined to patients with N0/1 disease $(P=0.008)$. Disease-free survival was significantly longer in the perioperative chemotherapy arm $(P=0.02)$, with a similar interaction in patients with N0/1 disease $(P=0.002)$. Risk of distant metastasis was significantly decreased in the group treated with preoperative chemotherapy (HR, 0.54; $P=0.01) .49$

Recently, several other neoadjuvant chemotherapy trials in the setting of resectable disease (Table 5) have been initiated. Unfortunately, the accrual of patients in these studies is slow and may further be compromised by the fact that a surgeryalone arm is considered unethical based on the results of the recently concluded adjuvant studies.

The Southwest Oncology Group (SWOG) S9900 trial was planned to assess whether preoperative chemotherapy with paclitaxel/carboplatin improves survival compared with surgery alone in previously untreated patients with stage IB, II, or selected stage IIIA (T3 N1) NSCLC. Operative mortality, response, and safety are evaluated. Samples will also be evaluated for molecular biologic factors and any correlation with outcome. Patients have been stratified on the basis of disease stage (IB/IIA vs. IIB/IIIA). The planned number of patients was set at 600 , but the trial closed on July 15, 2004, because of the previously described studies showing the efficacy of adjuvant chemotherapy in this patient population, making continued accrual to the control arm inappropriate.

Similar to the SWOG 9900, the Chemotherapy in Early Stages Trial (ChEST) evaluates the role of preoperative chemotherapy in patients with resectable stage IB, II, and selected IIIA (T3 N1) NSCLC, but the chemotherapy evaluated consists of gemcitabine $1000 \mathrm{mg} / \mathrm{m}^{2}$ on days 1 and 8 and cisplatin $75 \mathrm{mg} / \mathrm{m}^{2}$ on day 2 . Patients are randomized to immediate surgery followed by observation or induction gemcitabine/cisplatin for 3 cycles followed by surgery. Patient accrual was set at 700 and currently $>250$ patients are entered in the study. The primary endpoint is OS.
Figure 1 NATHL Treatment Schema

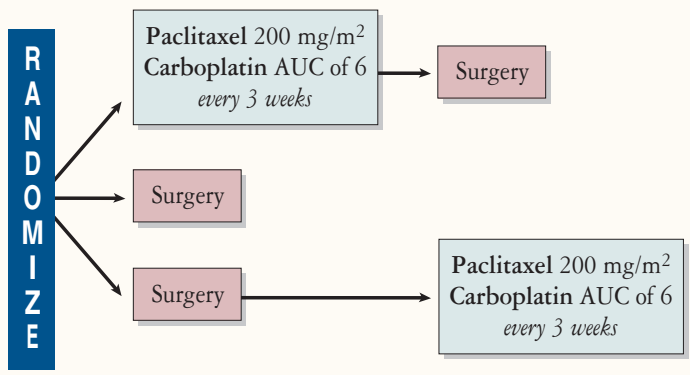

The Neoadjuvant Taxol ${ }^{\circledR}$ Carboplatin Hope (NATCH) trial compares the benefit of preoperative versus postoperative chemotherapy with paclitaxel/carboplatin versus surgery alone in stage IA $(>2.5 \mathrm{~cm}), \mathrm{IB}, \mathrm{II}$, and IIIA (T3 N1) NSCLC. Planned accrual was set at $>600$ patients. Paclitaxel $200 \mathrm{mg} / \mathrm{m}^{2}$ over 3 hours and carboplatin at an area under the curve of 6 are administered before surgery (arm 1) or after surgery (arm 3; Figure 1). Genetic polymorphisms for the xeroderma pigmentosum $\mathrm{D}$ gene at codons 751 and 312 were also evaluated for a correlation with response to treatment. Preliminary data about this trial are awaited in 2005.

Finally, a new French trial is currently evaluating the efficacy of 2 different regimens (cisplatin/gemcitabine and carboplatin/paclitaxel) and 2 different schedules ( 2 vs. 4 cycles of preoperative chemotherapy) in patients with stage I/II NSCLC. The expected number of patients is 130 in each arm for a total number of 520 patients. The primary endpoint is OS.

\section{Conclusion}

The most recent randomized studies of adjuvant chemotherapy testing the efficacy of the newer generation of cytotoxic agents in combination with platinum compounds have suggested a positive impact on efficacy outcomes (DFS and OS). A precise estimation of the survival gain that a metaanalysis estimated to be approximately $5 \%$ at 5 years needs to be finetuned. Two randomized clinical studies performed in highly selected patient populations support the use of adjuvant treatment in completely resected stage IB/II NSCLC. Two additional larger randomized clinical studies (one marginally positive and one marginally negative) in all stages of completely resected NSCLC indicate that, if any benefit from adjuvant chemotherapy exists, it is approximately $5 \%$ at 5 years. Another metaanalysis that will review the most recent generation of these positive and negative randomized clinical studies performed in the past 10 years will greatly contribute to assess more precisely the role of adjuvant chemotherapy.

Despite these encouraging results, reliable predictive and prognostic factors represent a priority to avoid the exposure of most patients to unnecessary treatments. In this perspective, genomics (or pharmacogenomics) and proteomics may in the near future drive the detection of those patients who are ideal candidates for adjuvant treatments. Although molecular-target- 
ed therapies appear in the adjuvant setting as a rationally designed approach, clinical validation of these "proof of principle" concepts through carefully designed clinical trials is absolutely mandatory.

\section{References}

1. Nesbitt JC, Putnam JB Jr, Walsh GL, et al. Survival in early-stage non-small cell lung cancer. Ann Thorac Surg 1995; 60:466-472.

2. Mountain CF. Revisions in the international system for staging of lung cancer. Chest 1997; 111:1710-1717.

3. Feld R, Rubenstein I, Weisenberger T, et al. Sites of recurrence in resected stage I nonsmall cell lung cancer: a guide for future studies. J Clin Oncol 1984; 2:1352-1358.

4. Martini N, Flehinger B, Zaman M, et al. Prospective study of 445 lung carcinomas with mediastinal lymph node metastases. J Thorac Cardiovasc Surg 1980; 80:390-397.

5. Pairolero P, Williams D, Bergstralh M, et al. Post-surgical stage I bronchogenic carcinoma. Morbid implications of recurrent disease. Ann Thorac Surg 1984; 38:331-338.

6. Thomas P, Rubenstein I. The Lung Cancer Study Group. Cancer recurrence after resection T1N0 non-small cell lung cancer. Ann Thorac Surg 1990; 48:242-247.

7. Speer JF, Petrosky VE, Retsky MW, et al. A stochastic numerical model of breast cancer growth that similates clinical data. Cancer Res 1984; 44:4124-4130.

8. PORT Meta-analysis Trialists Group. Postoperative radiotherapy in non-small-cell lung cancer: Systematic review and meta-analysis of individual patient data from nine randomized controlled trials. Lancet 1998; 352:257-263.

9. Machtay M, Lee JH, Shrager JB, et al. Risk of death from intercurrent disease is not excessively increased by modern postoperative radiotherapy for high-risk resected nonsmall cell lung carcinoma. J Clin Oncol 2001;19:3912-3917.

10. Mayer R, Smolle-Juettner FM, Szolar D, et al. Post-operative radiotherapy in radically resected non-small cell lung cancer. Chest 1997; 112:954-959.

11. Wagner $\mathrm{H} \mathrm{Jr}$, Bonomi P. Preoperative and postoperative therapy for non-small cell lung cancer. In: Roth JA, Ruckdeschel JC, Weisenburger TH, eds. Thoracic Oncology, 2nd ed. Philadelphia: WB Saunders, 1995:147-163.

12. Schabel FM Jr. Rationale for adjuvant chemotherapy. Cancer 1977; 39(suppl 6):28752882.

13. Holmes EC, Gail M. Surgical adjuvant therapy for stage II and stage III adenocarcinoma and large-cell undifferentiated carcinoma. J Clin Oncol 1986; 4:710-715.

14. Lad T, Rubinstein L, Sadeghi A. The benefit of adjuvant treatment for resected locally advanced non-small-cell lung cancer. J Clin Oncol 1988; 6:9-17.

15. Ohta M, Tsuchiya R, Shimoyama M, et al. Adjuvant chemotherapy for completely resected stage III non-small-cell lung cancer. J Thorac Cardiovasc Surg 1993; 106:703-708.

16. Figlin RA, Piantodosi S. A phase 3 randomized trial of immediate combination chemotherapy vs delayed combination chemotherapy in patients with completely resected stage II and III non-small cell carcinoma of the lung. Chest 1994; 106(6 suppl):310S-312S.

17. Dautzenberg B, Chastang C, Arriagada R, et al. Adjuvant radiotherapy versus combined sequential chemotherapy followed by radiotherapy in the treatment of resected non-small cell lung carcinoma. A randomized trial of 267 patients. Cancer 1995; 76:779-786.

18. Niiranen S, Niitamo-Korhonen S, Kouri M, et al. Adjuvant chemotherapy after radical surgery for non-small cell lung cancer: a randomized study. J Clin Oncol 1992; 10:1927-1932.

19. Feld R, Rubinstein L, Thomas PA. Adjuvant chemotherapy with cyclophosphamide, doxorubicin, and cisplatin in patients with completely resected stage I non-small-cell lung cancer. J Natl Cancer Inst 1993; 85:299-306.

20. Non-Small Cell Lung Cancer Collaborative Group. Chemotherapy in non-small cell lung cancer: a meta-analysis using updated data on individual patients from 52 randomized clinical trials. Br Med J 1995; 311:899-909.

21. Early Breast Cancer Trialists' Collaborative Group. Systemic treatment of early breast cancer by hormonal, cytotoxic and immune therapy. Lancet 1992; 339:1-15.

22. The International Adjuvant Lung Cancer Trial Collaborative Group. Cisplatin-based adjuvant chemotherapy in patients with resected non-small cell lung cancer. $N$ Engl J Med 2004; 350:351-360.

23. Scagliotti GV, Fossati R, Torri V, et al. Randomized study of adjuvant chemotherapy for completely resected stage I, II, or IIIA non-small-cell lung cancer. J Natl Cancer Inst 2003; 95:1453-1461.

24. Waller D, Fairlamb DJ, Gower N, et al. Determining the value of cisplatin-based chemotherapy for all patients with non-small cell lung cancer (NSCLC). Preliminary results in the surgical setting. Proc Am Soc Clin Oncol 2003; 22:632 (Abstract \#2543)
25. Strauss G, Herndon J, Maddaus M, et al. Randomized clinical trial of adjuvant chemotherapy with paclitaxel and carboplatin following resection in stage IB non-small cell lung cancer (NSCLC): report of Cancer and Leukemia Group B (CALGB) Protocol 9633. Late-breaking abstract from the 2004 ASCO Annual Meeting. J Clin Oncol 2004; 22(suppl):7019.

26. Winton T, Livingston R, Johnson $\mathrm{D}$, et al. A prospective randomized trial of adjuvant vinorelbine (VIN) and cisplatin (CIS) in completely resected stage IB and II non-small cell lung cancer (NSCLC) Intergroup JBR.10. Late-breaking abstract from the 2004 ASCO Annual Meeting. J Clin Oncol 2004; 22(suppl): 7018.

27. Keller SM, Adak S, Wagner H, et al. A randomized trial of postoperative adjuvant therapy in patients with completely resected stage II or IIIA non-small cell lung cancer. $N$ Engl J Med 2000; 343:1217-1222.

28. Crinò L, Clerici M, Figoli F, et al. Chemotherapy of advanced non-small cell lung cancer: a comparison of three active regimens. A randomized trial of the Italian Oncology Group for Clinical Research (GOIRC). Ann Oncol 1995; 6:347-353.

29. Martini N, Kris MG, Flehinger BJ, et al. Preoperative chemotherapy for stage IIIa (N2) lung cancer: the Sloan-Kettering Experience with 136 patients. Ann Thorac Surg 1993; 55:1365-1374.

30. Rosell R, Gomez-Codina J, Camps C, et al. A randomized trial comparing preoperative chemotherapy plus surgery with surgery alone in patients with non-small-cell lung cancer. N Engl J Med 1994; 330:153-158.

31. Roth JA, Fossella F, Komaki R, et al. A randomized trial comparing perioperative chemotherapy and surgery with surgery alone in resectable stage IIIA non-small-cell lung cancer. J Natl Cancer Inst 1994; 86:673-680.

32. Wu Y, Huang Z, Wang $S$, et al. A randomized trial of systematic nodal dissection in resectable non-small cell lung cancer. Lung Cancer 2002; 36:1-6.

33. Ambrogi V, Pompeo E, Elia S, et al. The impact of cardiovascular comorbidity on the outcome of surgery for stage I and II non-small cell lung cancer. Eur J Cardiovasc Surg 2003; 23:811-817.

34. Pastorino U, Valente M, Bedini V, et al. Effect of chronic cardiopulmonary disease on survival after resection for stage Ia lung cancer. Thorax 1982; 37:680-683.

35. Wu Y, Lin CJ, Hsu W, et al. Long-term results of pathological stage I non-small cell lung cancer: validation of using the number of totally removed lymph nodes as a staging system. Eur J Cardiovasc Surg 2003; 24:994-1001.

36. The Study Group of Adjuvant Chemotherapy for Lung Cancer: a randomised trial of postoperative adjuvant chemotherapy in non-small cell lung cancer. Eur J Surg Oncol 1995; 21:69-77.

37. Wada H, Hitami S, Teramatsu T, et al. Adjuvant chemotherapy after complete resection in non-small cell lung cancer. J Clin Oncol 1996; 14:1048-1054.

38. Wada H, Mivahara R, Tanaka F, et al. Postoperative adjuvant chemotherapy with PVM and UFT in resected stage I-II NSCLC: a randomised clinical trial. Eur J Cardiothorac Surg 1999; 15:438-443.

39. Endo C, Saito Y, Iwanami H, et al. A randomised trial of postoperative UFT therapy in stage I, II non-small cell lung cancer: North-East Japan Study group for Lung Cancer surgery. Lung Cancer 2003; 40:181-186.

40. Kato H, Ichinose Y, Ohta M, et al. A randomised trial of adjuvant chemotherapy with uracil-tegafur for adenocarcinoma of the lung. New Engl J Med 2004; 350:1713-1721.

41. Hamada C, Ohta M, Wada H, et al. Survival benefit of oral UFT for adjuvant chemotherapy after completely resected non-small cell lung cancer. Proc Am Soc Clin Oncol 2004; 23:615 (Abstract \#7002).

42. Fukuoka M, Yano S, Giaccone G, et al. Multi-institutional randomized phase II trial of gefitinib for previously treated patients with advanced non small cell lung cancer. $J$ Clin Oncol 2003; 21:2237-2246.

43. Kris MG, Natale RB, Herbst RS, et al. Efficacy of gefitinib, an inhibitor of the epidermal growth factor receptor tyrosine kinase, in symptomatic patients with non-small cell lung cancer: a randomised trial. JAMA 2004; 290:2149-2158.

44. Hirsch FR, Franklin WA, Gazdar AF, et al. Early detection of lung cancer: clinical perspectives of recent advances in biology and radiology. Clin Cancer Res 2001; 7:5-22.

45. Slebos RJC, Hruban RH, Dalesio O, et al. K-ras oncogene activation as a prognostic marker in adenocarcinoma of the lung. N Engl J Med 1990; 323:561-565.

46. Huncharek M, Muscat J, Geschwind JF. K-ras oncogene mutation as a prognostic marker in non-small cell lung cancer: a combined analysis of 881 cases. Carcinogenesis 1999; 20:1507-1510.

47. Scagliotti GV, Masiero P, Pozzi E. Biological prognostic factors in non-small cell lung cancer. Lung Cancer 1995; 12 (suppl 1):S13-S25.

48. Pastorino U, Andreola S, Tagliabue E, et al. Immunocytochemical markers in stage I lung cancer: relevance to prognosis. J Clin Oncol 1997; 15:2858-2865.

49. De Pierre A, Milleron B, Moro-Sibilot D, et al. Preoperative chemotherapy followed by surgery compared with primary surgery in resectable stage I (except T1N0), II and IIIa non-small cell lung cancer. J Clin Oncol 2002; 20:247-253. 